Dobell, A. R.

"A Comment on A. Y. C. Koo's "An Empirical Test of Revealed Preference Theory"." Econometrica 33.2 (1965): 451-455.

\author{
Reprinted with permission from \\ The Econometrics Society
}

The copyright to this article is held by the Econometric Society, http:// www.econometricsociety.org/ . It may be downloaded, printed and reproduced only for personal or classroom use. Absolutely no downloading or copying may be done for, or on behalf of, any for-profit commercial firm or other commercial purpose without the explicit permission of the Econometric Society. For this purpose, contact Claire Sashi, General Manager, at sashi@econometricsociety.org. 


\title{
A COMMENT ON A. Y. C. KOO'S “AN EMPIRICAL TEST OF REVEALED PREFERENCE THEORY”
}

\author{
By A. R. DobelL ${ }^{1}$
}

THIS NOTE POINTS out an objection to Koo's paper [3] and suggests a possible modification, based on some standard results in graph theory.

It is desirable to review briefly some background before turning to Koo's paper itself. The "theory of revealed preference" is customarily developed from a binary relation $R$ defined on a commodity space, and an axiom (S) governing this relation, as follows.

Denote by $q^{i}$ a point (called a goods bundle) in a $k$-dimensional Euclidean space (called the commodity space) and by $p^{i}$ the price vector at which the goods bundle $q^{i}$ is selected by a consumer. Denote by $p \cdot q$ the inner product of two vectors $p$ and $q$.

DEFINITION: The binary relation $R$ ("revealed preferred") is defined by the condition $q^{0} R q^{1} \Leftrightarrow p^{0} \cdot q^{0} \geqslant p^{0} \cdot q^{1}, q^{1} \neq q^{0}$. That is, a goods bundle $q^{0}$ is revealed preferred to a distinct goods bundle $q^{1}$ if $q^{1}$ could have beon purchased at prices $p^{0}$ but was rejected in favor of $q^{0}$.

Axiom (S): For any positive integer $n$,

$$
q^{0} R q^{1}, q^{1} R q^{2}, \ldots, q^{n-1} R q^{n} \Rightarrow \sim\left(q^{n} R q^{0}\right),
$$

where the symbol " " denotes negation. That is, if there are distinct goods bundles $q$ such that $q^{i}$ is revealed preferred to $q^{i+1}$ for $i=0,1,2, n-1$, then $q^{n}$ cannot be revealed preferred to $q^{0}$.

The axiom (S) is the "strong axiom of revealed preference" formulated by Houthakker [2]; the special case in which the condition is imposed only for $n=1$ is known as the "weak axiom of revealed preference," and was employed by Samuelson in his pioneering article [6]. The primary purpose of the theory built on these concepts is to demonstrate that from the axiom (S) on individual demand functions (assumed to be single-valued and "smooth"), the observable "laws of demand" can be directly derived.

To test the validity of this approach to consumers' behavior, one might examine the purchases of a consumer to determine whether they were in fact consistent with the axiom (S). Suppose then that one had $n$ market observations for a single consumer, i.e., had recorded $n$ distinct goods bundles $q^{i}$ purchased in response to

${ }^{1}$ I wish to thank Professors P. A. Samuelson and F. M. Fisher for their comments on this note. 
prices $p^{i}$, for $i=1,2, \ldots, n$. All relevant information can be summarized in a matrix

$$
A=\left[a_{i j}\right]=\left[p^{i} \cdot q^{j}\right] .
$$

It is convenient to form the matrix

$$
B=\left[b_{i j}\right]=\left[a_{i j}-a_{i i}\right]
$$

by subtracting the diagonal element of each row from every element of the row. Finally, since only orderings are relevant, one can equally well use the matrix

$$
C=\left[c_{i j}\right], \text { where } c_{i j}=\left\{\begin{array}{l}
1 \text { if } b_{i j} \leqslant 0, i \neq j ; \\
0 \text { otherwise }
\end{array}\right.
$$

Thus, if $c_{i j}=1$, this means $a_{i j} \leqslant a_{i i}, i \neq j$, or in other words $q^{i} R q^{j} .^{2}$

By examination of this matrix $C$, one can determine whether the observed market data show any violation of the axiom (S). Three cases might be distinguished:

(1) The market data suffice to show a complete ordering of all $n$ of the goods bundles.

(2) The data show a violation of the axiom (S).

(3) Neither (1) nor (2).

From the construction of the matrix $C$, it is clear that a complete ranking of the $n$ goods bundles can be deduced on the basis of the market data alone (that is, case (1) can obtain) if and only if the matrix $C$ can be put, by suitable renumbering of goods bundles, into a strictly triangular form, with zero's on and above the diagonal, one's below. Professor Koo concentrates on this case and offers [3, p. 654] a condition characterizing matrices falling in this category-namely that they must have a row and a column having exactly $i$ one's for each value of $i=0,1,2$, $\ldots, n-1 .^{3}$ But this property is neither necessary nor sufficient for consistency of

${ }^{2}$ Matrices like $C$ occur frequently in connection with graph theory or the study of binary relations. To any binary relation $R$ on a finite set of elements, for example, there corresponds a matrix in which the $i j$ th entry is 1 if and only if element $i$ stands in relation $R$ to element $j$. (One notes that the matrix $C$ above is exactly the matrix which corresponds by this construction to the relation "revealed preferred" on the set $\left\{q^{1} \ldots q^{n}\right\}$.) Alternatively, one may also construct a directed graph corresponding to $R$ by representing the elements as points in the plane, and drawing a directed arc from point $i$ to point $j$ if and only if the $i$ th element stands in relation $R$ to the $j$ th. Thus one has two equivalent representations of a binary relation; the matrix form is frequently convenient because it lends itself readily to automatic computation. In such use, the matrices may -for computational convenience-be considered to be Boolean matrices-that is, matrix addition and multiplication are defined by the usual formulas, but arithmetic is governed by Boolean algebra so that $1+1=1$. Thus, the sum or product of two Boolean matrices is again a Boolean matrix. Some useful features of these concepts will be indicated later. Detailed study and references can be found in [1].

${ }^{3}$ The matrix with which Professor Koo works is almost $C$, except that if $C$ has a pair of symmetric elements both one's, he replaces these by zero's. For this discussion the difference does not matter. 
consumer behavior (that is, for the validity of (S)); it is a reflection solely of the happy chance that yielded the particular set of market observations.

What would seem to be a more interesting question is whether or not the matrices $C$ show any violation of the axiom (S). This question can be tested by appeal to the following theorem, recalling that a matrix $A$ is said to be nilpotent if there exists a positive integer $m$ such that $A^{m}$ is the zero matrix.

THEOREM: A necessary condition for $n$ market observations to be consistent with (S) is that the associated Boolean matrix $C$ be nilpotent; for this it is necessary that every principal submatrix of $C$ have at least one zero row and zero column. ${ }^{4}$

Turning the theorem around, a sufficient condition that the $n$ observations show a violation of (S) is that the matrix $C$ not satisfy the above requirement. One can test this simply by deleting from $\{q\}$ a goods bundle associated with a zero row or column, and constructing the principal submatrix of $C$ corresponding to the reduced set. This principal submatrix is then examined for zero rows or columns, and the process continued. If the entire set $\{q\}$ cannot be so deleted, there is a violation of (S). Such a test is easily programmed.

The restricted nature of Koo's result is then clear. It is true that his triangular matrix with zero's on the diagonal is nilpotent-this is a special case in which each principal submatrix has exactly one zero row and one zero column. But clearly many other cases are compatible with (S).

Revealed preference theory is primarily a direct derivation of the laws of demand from the hypothesis (S). Koo's "test" of the theory is not a test in the usual sense of confronting this hypothesis with data in order possibly to refute the hypothesis. Rather his results indicate only how helpful market observations might be in constructing directly the consumer's utility index. It is true that Samuelson [7] and Houthakker [2] have shown that with sufficiently many market observations one can-if (S) holds-in principle construct an ordinal utility function for a consumer. But nothing in the theory falls if we happen to be unlucky in our first batch of observations. Nor can the information that one consumer's "maximal consistent subset" [3, p. 654] is larger than another's lead to any conclusion regarding the consistency of one as compared to the other.

The above comments can be illustrated by brief mention of Koo's introductory examples. He states the "test of consistency in its general form" as

$$
\text { “. . It is impossible that } p^{0} \cdot q^{0}>p^{0} \cdot q^{1} \text { and } p^{1} \cdot q^{0}<p^{1} \cdot q^{1} . " 5
$$

4 This theorem is stated by Marimont $[4,5]$ and used in the study of precedence relationships in computer programming. Its application in the present problem is evident when one observes that a violation of (S) amounts to the presence of a closed cycle in the directed graph corresponding to $C$, and that requiring $C$ to be nilpotent is simply a way of ensuring that $C$ be acyclic.

$5[3$, p. 647]. The difference between the strict inequalities required here and the equalities permitted in (S) does not matter in this discussion. 
He then offers three illustrations, as follow:

$$
\text { Example (1). } p^{0} \cdot q^{0}>p^{0} \cdot q^{1} ; p^{1} \cdot q^{0}>p^{1} \cdot q^{1} \text {. }
$$

This is the case in which the consumer's preferences between two goods bundles are unambiguously revealed by only two market observations. (The corresponding matrix $C$ is $C=\left[\begin{array}{ll}0 & 1 \\ 0 & 0\end{array}\right]$, which has the required strictly triangular form, with $C^{2}=0$.)

Example (2). $p^{0} \cdot q^{0}>p^{0} \cdot q^{1} ; p^{1} \cdot q^{0}<p^{1} \cdot q^{1}$.

This example Koo correctly identifies as inconsistent with $\mathbf{S}^{\prime}$. (The corresponding matrix $C=\left[\begin{array}{ll}0 & 1 \\ 1 & 0\end{array}\right]$ is cyclic and therefore not nilpotent.)

Example (3). $p^{0} \cdot q^{0}<p^{0} \cdot q^{1} ; p^{1} \cdot q^{0}>p^{1} \cdot q^{1}$.

(The matrix $C=\left[\begin{array}{ll}0 & 0 \\ 0 & 0\end{array}\right]$.) This example is not correctly identified by Koo; he asserts that it "fails to satisfy the consistency test" [3, p. 649]. In fact, however, this example is a completely valid instance of the proposition $\left(\mathbf{S}^{\prime}\right)$; in no way is it incompatible with the hypothesis of consistent consumer behavior. The only condition Example (3) fails to meet is that two market observations be enough to identify the consumer's ranking of the two goods bundles. But, as mentioned above, this requirement has nothing to do with the logical structure of revealed preference theory, and a failure to meet this requirement in no way constitutes a failure of the theory.

Thus, in summary, Professor Koo's procedure can be interpreted essentially as that described at the beginning of this note, down to the construction of the matrix $C$, and identification of case (1). Because it is confined entirely to this case, Professor Koo's test, as it stands, tests neither the hypothesis $\left(\mathbf{S}^{\prime}\right)$ nor the consistency of a consumer, but only his own luck in acquiring really revealing market data.

I have therefore suggested a simple extension which does provide the basis for an easily programmed test of consistency with the hypothesis of revealed preference theory, and have indicated the close connection between such methods and some standard results of graph theory. Such an extension is necessary even to render Professor Koo's procedure valid in principle.

\section{Massachusetts Institute of Technology}

\section{REFERENCES}

[1] Flament, Claude: Applications of Graph Theory to Group Structure, Prentice Hall, Englewood Cliffs, 1963.

[2] Houthakker, H. S.: "Revealed Preference and the Utility Function," Economica, n.s., 17 (May, 1950), pp. 159-174. 
[3] Koo, A. Y. C.: “An Empirical Test of Revealed Preference Theory,” Econometrica, 31 (October, 1963), pp. 646-664.

[4] Marimont, R. B.: "Applications of Graphs and Boolean Matrices to Computer Programming," SIAM Review, 2 (October, 1960), pp. 259-268.

[5] ___ "A New Method of Checking the Consistency of Precedence Matrices," Journal of the Association for Computing Machinery, 6, 1959, pp. 164-171.

[6] Samuelson, P. A.: “A Note on the Pure Theory of Consumer's Behavior," Economica, n.s., 5 (February, 1938), pp. 67-71.

[7] —_: "Consumption Theory in Terms of Revealed Preference," Economica, n.s., 15 (November, 1948), pp. 243-253. 